\title{
Different hyaluronic acid morphology modulates primary articular chondrocyte behavior in hyaluronic acid-coated polycaprolactone scaffolds
}

\author{
Myriam Lebourg, ${ }^{1}$ Joaquin Rodenas Rochina, ${ }^{2}$ Tiago Sousa, ${ }^{2,3}$ \\ João Mano, ${ }^{3,4}$ José Luis Gómez Ribelles ${ }^{1,2}$ \\ ${ }^{1}$ CIBER de Bioingeniería, Biomateriales y Nanomedicina (CIBER-BBN), Valencia, Spain \\ ${ }^{2}$ Center for Biomaterials and Tissue Engineering, Universitat Politècnica de València, Camino de Vera s/n, \\ 46022 València, Spain \\ ${ }^{3} 3$ Bs Research Group-Biomaterials, Biodegradables, and Biomimetics University Minho, Headquarters of the European \\ Institute of Excellence on Tissue Engineering and Regenerative Medicine, AvePark, S. Claúdio do Barco 4806-909 Taipas, \\ Guimarães, Portugal \\ ${ }^{4}$ ICVS/3Bs-PT Government Associate Laboratory, Braga/Guimarães, Portugal
}

Received 27 December 2011; revised 4 June 2012; accepted 20 June 2012

Published online 28 August 2012 in Wiley Online Library (wileyonlinelibrary.com). DOI: 10.1002/jbm.a.34349

\begin{abstract}
Scaffolds for cartilage tissue engineering should promote both adequate biomechanical environment and chondrogenic stimulation. Hyaluronic acid (HA) has been used in cartilage engineering for its chondrogenic and chondroprotective properties, nevertheless its mechanical properties are limited. Influence of HA microstructure in chondrocyte response has not been addressed yet. In this work, polycaprolactone (PCL) scaffolds were modified using HA following two coating strategies: coating in one step (PCL-HA1s) yields a gel-like phase within the scaffold, whereas a two-step reaction (PCL-HA2s) yields a thin HA layer coating internal surfaces of PCL structure. Chondrocytes were seeded in the scaffolds and cultured in dedifferentiating conditions up to 3 weeks and analyzed using a total DNA assay and sulfated glycosaminoglycan (sGAG) determination assay; cell morphology and extracellular matrix secretion were assessed by electron microscopy as well as immuno-
\end{abstract}

fluorescent imaging (collagen I, collagen II, aggrecan, CD44). Cells proliferate in all samples and no cytotoxicity is observed. PCL-HA1s shows higher sGAG production per cell than PCL and PCL-HA2s at all times. Presence of hyaluronic acid promotes qualitative expression of CD44 surface markers and aggrecan (more visible in PCL-HA1s than PCL-HA2s), whereas in dedifferentiating conditions, expression of CD44 and aggrecan can hardly be detected in pure PCL scaffolds. Collagen type II seems more prominent in PCL-HA2s; although PCLHA2s shows markers for COL II, aggrecan and CD44, quantitative ECM production is not improved with respect to PCL. It is thus likely that CD44 activation is not sufficient for explaining the better response in PCL-HA1s. (c) 2012 Wiley Periodicals, Inc. J Biomed Mater Res Part A: 101A: 518-527, 2013.

Key Words: polycaprolactone, tissue engineering, cartilage, human chondrocyte

How to cite this article: Lebourg M, Rochina JR, Sousa T, Mano J, Ribelles JLG. 2013. Different hyaluronic acid morphology modulates primary articular chondrocyte behavior in hyaluronic acid-coated polycaprolactone scaffolds. J Biomed Mater Res Part A 2013:101A:518-527.

\section{INTRODUCTION}

Articular cartilage repair is a challenge for tissue engineering, as cartilage has a very limited capacity for repair, due to the lack of vascularization, the low cellularity of the tissue and the limited anabolic capacity of chondrocytes. ${ }^{1,2}$ Autologous chondrocyte implant has been implemented in clinical practice for a decade, with promising results in patients with single lesions. ${ }^{3}$ Shortcomings include the need for extensive in vitro cell expansion, which induces dedifferentiation of chondrocytes, ${ }^{4-6}$ the difficulty to apply the technique to extensive defects, and the need of using a periosteal flap to maintain the cell pellet in the defect. This technique also implies complicated surgery procedures, and two operations are necessary, one to gather biopsy and another to

Additional Supporting Information may be found in the online version of this article.

Correspondence to: M. Lebourg; e-mail: myle1@upvnet.upv.es

Contract grant sponsor: Valencia Polytechnic University; contract grant number: PAID-06-10

Contract grant sponsor: Spanish Ministry of Science; contract grant number: MAT2010-21611-C03-01

Contract grant sponsors: CIBER-BBN; VI National R\&D\&i Plan 2008-2011; Iniciativa Ingenio 2010; Consolider Program; Instituto de Salud Carlos III; European Regional Development Fund; Valencian Generality; Conselleria de Sanidad (Generalitat Valenciana) 
implant the cell pellets). ${ }^{7}$ Furthermore, the lack of mechanical scaffolding may be a drawback due to the poor mechanical properties of the cell pellet and could cause damage and necrosis in the surrounding zone arising from stress concentration. As a matter of fact, poor integration of repair tissue with surrounding tissue remains a problem in existing cartilage therapies. ${ }^{8}$ Scaffolds are useful as carriers for implanted cells and eventually allow matching the biomechanical properties of native cartilage. A wide range of materials have been considered for articular cartilage tissue engineering, ranging from natural polysaccharides such as agarose, chitosan, hyaluronic acid, and their derivatives, as well as synthetic materials like poly(lactic-co-glycolic)acid, ${ }^{9}$ polylactic acid, polycaprolactone (PCL), and polyurethane. PCL scaffolds have been successfully used for repairing chondral defects in a rabbit model ${ }^{10}$; its elastic nature and low resorption rate allow for long term mechanical withstanding of the repair tissue. Nevertheless, PCL is a synthetic material that does not provide specific signals (e.g., biological interaction sites) that may enhance chondrocyte phenotype maintenance and proper extracellular matrix (ECM) secretion. ${ }^{11,12}$

On the other hand, hyaluronic acid (HA) is recognized by chondrocytes surface receptor CD44 and has been shown to have a positive influence on a large number of cellular pathways including chondrocyte proliferation, ${ }^{13}$ ECM secretion, ${ }^{14}$ and phenotypic regulation. ${ }^{15,16} \mathrm{HA}$ has also shown protective properties in case of osteoarthritic condition ${ }^{15}$ and oxidative stress of chondrocytes, ${ }^{17}$ being a recognized treatment as injectable liquid, as well in a gel state, after esterification, for implants $\left(\right.$ Hyaff $^{\circledR}$ ). The Hyaff $^{\circledR}$ clinical outcome is quite satisfying in young patients with single lesions, nevertheless it does not seem to yield significant improvement of patient wellbeing ${ }^{7}$ in more complicated cases. Rapid degradation of such scaffolds and intrinsically poor mechanical properties could compromise the biomechanical environment and be insufficient to obtain a whole regeneration of complicated defects before the scaffolding material disappears. ${ }^{18}$

The combination of a macroporous scaffold made of a polymer providing the desired mechanical properties and a HA coating of the pore walls can take advantage of the bioactive properties of HA while providing the seeded chondrocytes with an adequate biomechanical environment. In a previous study, physical properties of polylactide scaffolds coated with glutaraldehyde crosslinked HA were studied. ${ }^{19}$

Here we investigate hybrid scaffolds that show higher porosity, better defined pore architecture and slower degradation rate to evaluate their suitability for cartilage tissue engineering.

We hypothesize that the presence of HA will boost the performance of human primary articular chondrocytes when seeded in vitro in standard culture conditions and inhibit the dedifferentiation that is observed when culturing cells with fetal bovine serum in PCL scaffolds ${ }^{23}$; we further hypothesize that the physical structure of hyaluronic acid in the hybrid scaffold has an outstanding importance in modulating cell response.

\section{MATERIALS AND METHODS} Scaffold preparation

Scaffolds were prepared by a mixed particle leaching/ freeze extraction process. Low molecular weight polyethylmetha- crylate (PEMA) beads (from Lucite International) with mean diameter $200 \mu \mathrm{m}$ were used as a porogen. Freeze extraction is a modification of freeze drying as proposed by Wang and coworkers. ${ }^{21}$ Here dioxane was used as a solvent for PCL. The PCL solution $(20 \% \mathrm{w} / \mathrm{w}$ in dioxane $)$ was mixed with PEMA beads in a weight ratio $1: 1$ and immediately frozen in liquid nitrogen. Solvent extraction was performed in cold ethanol at $-20^{\circ} \mathrm{C}$ as described elsewhere ${ }^{20}$; ethanol was changed three times. Subsequently particle leaching was performed in ethanol at $40^{\circ} \mathrm{C}$ until no more porogen was detected in the washing solvent.

\section{Coating in one step}

For the crosslinking in one step (PCL-HA1s), 2\% hyaluronic acid (from Streptococcus Equi, Sigma-Aldrich) was dissolved in $0.2 \mathrm{M}$ sodium hydroxide (Scharlau) aqueous solution under mild stirring.

The scaffolds were immersed in reaction vessels containing HA solution mixed with a 2:1 molar ratio of divinylsulfone (DVS). The vessels were then connected to a vacuum pump for $2 \mathrm{~min}$ to force the viscous HA solution to penetrate into the scaffold's pores, and allowed to react for $2 \mathrm{~h}$ at room temperature before washing. Scaffolds were washed with acetone/water mixture 50/50 and dried.

\section{Coating in two steps}

For the crosslinking in two steps (PCL-HA2s), 1\% hyaluronic acid was dissolved in distilled water under mild stirring at room temperature. During the first step, the scaffolds were immersed in the mentioned solution. The reactor vessels were connected to a vacuum pump for $2 \mathrm{~min}$ to force penetration of HA solution into the scaffold's pores; then excess HA outside of the sample was eliminated, and samples were allowed to dry in an oven at $37^{\circ} \mathrm{C}$ for 1 day and finally dried under vacuum. This process was repeated three times. Then in a second step, crosslinking was performed in acetone/water mix (80/20) at $\mathrm{pH} 12$ to prevent dissolution of HA but to permit some swelling and the necessary deprotonation of hydroxyl moieties of $\mathrm{HA}^{23}$ adding a 2:1 molar ratio of DVS with respect to reactive moieties. At the end of the reaction, samples were washed with $80 / 20$ (v/v) acetone/water mixture and allowed to dry at air.

\section{Morphology}

The morphology of the cross section of the coated scaffolds was analyzed using scanning electron microscopy (SEM) to analyze the shape of dry samples and CryoSEM to observe the samples swollen in water.

For observation with SEM, samples were cryofractured (to preserve the microstructure of the scaffolds) and mounted on copper stubs with a graphite conductive tape, and gold sputtered.

The microscope used was JEOL JSM6300 scanning electron microscope at an acceleration voltage of $15 \mathrm{kV}$.

For CryoSEM, wet samples were carefully wiped with filter paper, mounted in a clamp, ultrafrozen, and then cryofractured; the sublimation temperature used was $-50^{\circ} \mathrm{C}$. Finally samples were gold sputtered in the same cold 
vacuum chamber of a JEOL JSM6300 electron microscope and observed at an acceleration tension of $15 \mathrm{kV}$.

\section{Compression test}

Compression tests were performed using a Microtest universal testing machine with $15 \mathrm{~N}$ load cell on cylindrical samples of $6 \mathrm{~mm}$ diameter and around $5 \mathrm{~mm}$ in height. A displacement ramp was applied at $1 \mathrm{~mm} \mathrm{~min}{ }^{-1}$ until a force of $14 N$ was reached. Elastic compressive modulus of the scaffold $(E)$ was determined as the slope of the linear elastic zone of a stress-strain representation (see Supporting Information Fig. 1). Methodology for curve interpretation and modulus calculation was taken from American Society for Testing and Materials D1621-04a standard "compressive properties of rigid cellular plastics." The results presented are average values of five measurements; error bars correspond to standard deviation.

\section{Water absorption behavior}

For water absorption analysis samples were immersed in distilled water and allowed to take up water until equilibrium was reached $(48 \mathrm{~h})$. Samples were weighed dry $\left(W_{\mathrm{d}}\right)$ and wet $\left(W_{\mathrm{s}}\right)$ and a ratio describing water uptake was calculated according to Eq. (1):

$$
\text { Swelling ratio }=\frac{W_{\mathrm{s}}}{W_{\mathrm{d}}}
$$

Five samples were measured for each group.

\section{Thermogravimetric analysis}

The thermogravimetric analysis was performed in a SDT Q6000 thermogravimetric analyzer (TGA) from TA Instruments to determine the amount of crosslinked HA present in both types of coated scaffolds.

The samples were placed in a platinum pan and subjected to a heating scan from 50 to $1000^{\circ} \mathrm{C}$ at $20^{\circ} \mathrm{C} \mathrm{min}^{-1}$ under nitrogen atmosphere.

The mass was monitored as a function of the temperature; results were analyzed using the software TA analyzer from the instrument. Degradation peaks of HA and PCL were determined using bare crosslinked HA and PCL samples: using differential weight loss signal, onset and offset temperatures of the first degradation peak associated to HA at $T=255^{\circ} \mathrm{C}$ were determined. From the weight scan of pure $\mathrm{HA}$, it has been determined that the weight loss at this temperature corresponds to $35 \%$ of the total mass of HA. Weight loss percentage between these temperatures was determined, and corresponding HA weight in the sample was calculated (see Supporting Information Fig. 2).

\section{Cell culture}

Prior to cell culture experiments, cylindrical samples of $6 \times$ $5 \mathrm{~mm}^{2}$ were washed in phosphate saline buffer (PBS), then sterilized in ethanol for $3 \mathrm{~h}$ at $4^{\circ} \mathrm{C}$, washed several times in PBS and placed in a multiwell tissue-culture polystyrene plate with high glucose medium supplemented with $1 \%$ penicillin/streptomycin and 10\% fetal bovine serum (FBS) to permit adsorption of serum proteins onto the scaffolds.

Human chondrocytes were harvested from a knee of a patient undergoing arthroplasty (man, 71 years) after informed consent following the guidelines of the ethical committees of Universitat Politècnica and Clínica de la Salud of Valencia. The cartilage was dissected from subchondral bone, finely diced and then washed with supplemented 100 $\mathrm{U}$ penicillin, $100 \mu \mathrm{g}$ streptomycin (Biological Industries) Dulbecco's modified Eagle's medium (DMEM) (Gibco). For chondrocyte isolation, finely diced cartilage was incubated for $30 \mathrm{~min}$ with $0.5 \mathrm{mg} \mathrm{mL} \mathrm{m}^{-1}$ hyaluronidase (SigmaAldrich) while shaking at $37^{\circ} \mathrm{C}$, and then with $1 \mathrm{mg} \mathrm{mL}{ }^{-1}$ pronase (Merck, VWR International SL) during $60 \mathrm{~min}$ in the same conditions. Subsequently the cartilage pieces were washed with supplemented DMEM and digested with 0.5 $\mathrm{mg} \mathrm{mL} \mathrm{m}^{-1}$ of collagenase-IA (Sigma-Aldrich) in a shaking water bath at $37^{\circ} \mathrm{C}$ overnight. The resulting cell suspension was filtered through a 70- $\mu \mathrm{m}$ pore nylon filter (BD Biosciences) to remove tissue debris. Cells were centrifuged and washed with DMEM supplemented with 10\% FBS (Invitrogen SA). Finally, the cells were plated in tissue culture flasks and amplified in a monolayer culture using T150 $\mathrm{cm}^{2}$ culture flasks until passage 2 for 10-12 days, in DMEM medium with $4.5 \mathrm{~g} \mathrm{~L}^{-1}$ Glucose, enriched with $10 \%$ of FBS, $1 \%$ penicillin/streptomycin, $1 \%$ of ascorbic acid, $1 \%$ of $\mathrm{D}-\mathrm{L}$ glutamine and $1 \%$ of L-pyruvate.

After trypsinization and cell counting following standard techniques, $\sim 4 \times 10^{5}$ cells suspended in DMEM $(50 \mu \mathrm{L}$ sample ${ }^{-1}$ ) were injected into the scaffolds with a syringe. The cells were allowed to adhere for $30 \mathrm{~min}$ in the incubator at $37^{\circ} \mathrm{C}$ and $5 \% \mathrm{CO}_{2}$ and then samples were put in new wells that were completed with culture medium at $500 \mu \mathrm{L}$ well $^{-1}$ (DMEM $4.5 \mathrm{~g} \mathrm{~L}^{-1}$ glucose, supplemented with $10 \%$ FBS, $1 \%$ penicillin/streptomycin, $1 \%$ ascorbic acid, 1\% D-L glutamine and $1 \%$ L-pyruvate). Two scaffolds of each type were left empty to be used as acellular controls for quantitative biochemical tests.

The culture was led in static conditions in an incubator at $37^{\circ} \mathrm{C}$ and $5 \% \mathrm{CO}_{2}$. The culture medium was changed every 2 days and samples were collected at 7, 14, and 21 days.

After being washed in Dulbecco phosphate saline buffer (DPBS), samples were fixed in $3.7 \%$ paraformaldehyde (Sigma-Aldrich) at $4^{\circ} \mathrm{C}$ for $1 \mathrm{~h}$. Samples for quantitative biochemical analysis were washed with DPBS and placed in centrifuge microtubes containing milliQ-water (Millipore) and stored in $\mathrm{a}-80^{\circ} \mathrm{C}$ freezer until analysis.

\section{Immunofluorescent staining}

For immunostaining, sample embedding and cutting was performed as follows: samples cut in halves were first immersed in a $30 \%$ sucrose solution overnight; then the scaffolds were taken out, lightly wiped to remove excess solution, placed in a mould with (OCT) optimum cutting temperature compound (OCT, Tissue Tek) and frozen rapidly at $-80^{\circ} \mathrm{C}$. The embedded scaffolds were cut longitudinally using the cryotome Microm HM 500 at $-30^{\circ} \mathrm{C}$ in $200-\mu$ m-thick sections, which were then placed over treated microscope slides (StarFrost). 


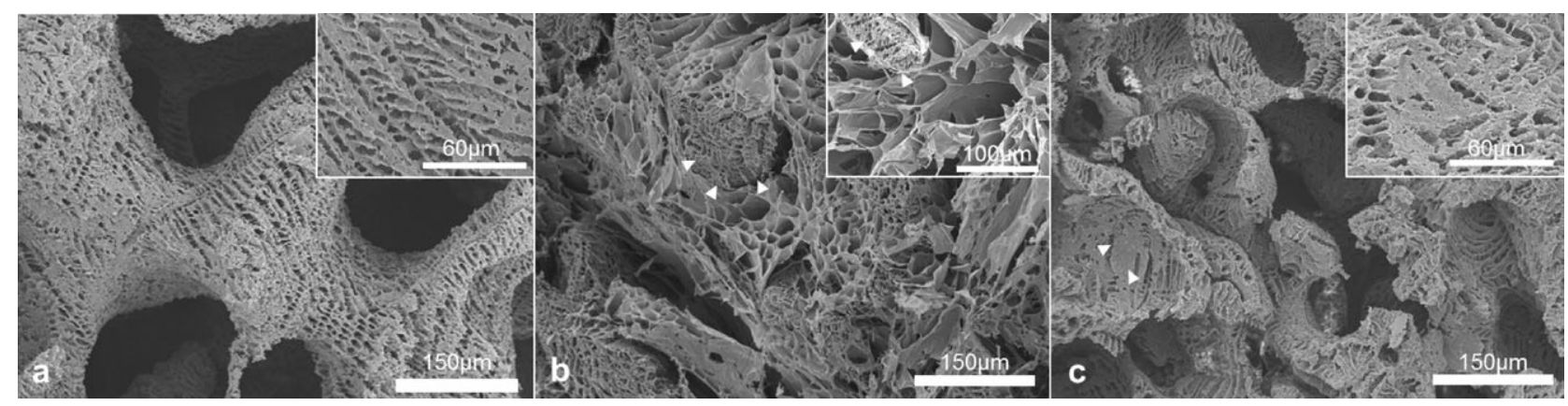

FIGURE 1. SEM picture of bare PCL-HA scaffold (a) and CryoSEM micrographs of HA-coated scaffolds previously swollen in water: PCL-HA1s (b) and PCL-HA2s (c). While PCL-HA2s structure is left empty and only a thin coating is seen (see detail with white arrows on picture 1b where the coating appears to hide micropore structure), PCL-HA1s is filled with a gel phase that surrounds the PCL scaffold (white arrows, 1a).

Sections were washed gently with DPBS $1 \times$ two times to eliminate the OCT. Then immunostaining was carried out following standard procedure. On the one side, staining for cell morphology and presence of proteoglycan was achieved using Phalloidin Bodipy FL (1/200, Invitrogen) for staining of actin and mouse anti-human aggrecan (1/50, Invitrogen) and Alexa 647 anti-mouse (1:400, Invitrogen) as a secondary antibody. On the other hand, staining of collagen type I and II was achieved using Rabbit $\alpha$-human collagen I antibody (1:50, Chemicon International) mixed with mouse $\alpha$-human collagen II antibody (1:100, Chemicon International) and using Alexa $488 \alpha$-rabbit (1:400, Invitrogen) and Alexa 647 $\alpha$-mouse (1:400, Invitrogen) as secondary antibodies. The presence of CD 44 receptors at the end of culture time was checked by staining with mouse anti-CD44 (1:200, Abcam) and Alexa $488 \alpha$-mouse (1:400, Invitrogen). Primary antibodies were incubated for $2 \mathrm{~h}$, secondary antibodies and Phalloidin for $1 \mathrm{~h}$. Finally, nuclei were stained with DAPI $(0.2 \mu \mathrm{g}$ $\mathrm{mL}^{-1}$, Sigma-Aldrich), and mounted with Fluorsave Vectashield mounting medium (Atom).

As a result of immunostaining, actin, collagen $\mathrm{I}$, and CD44 are stained green while aggrecans and collagen II are stained red. Nuclei are stained blue.

Immunostained sections were observed in a Leica IM 500 confocal microscope; for each sample 12 stacks of $3-\mu \mathrm{m}$ each were observed (samples were observed up to a depth of $30 \mu \mathrm{m})$.

\section{Sulfated glycosaminoglycans content}

The content of sulfated glycosaminoglycans (sGAG) was determined using Blyscan assay kit (modified version of dimethyl methylene blue assay) (Biocolor). In brief, samples were digested enzymatically using proteinase $\mathrm{K}$ (Roche). Thereafter, an aliquot of supernatant was reacted with Blyscan dye for $30 \mathrm{~min}$ protected from light on an orbital shaker. sGAG-dye precipitate was obtained by centrifugation and the resulting pellet was dissolved in $1 \mathrm{~mL}$ dissociation reagent and incubated for $10 \mathrm{~min}$ in an orbital shaker. Finally, samples were transferred to a 96-well-plate and absorbance was read in triplicate at $656 \mathrm{~nm}$ in a Victor microplate reader (Perkin Elmer). The quantities of sGAG were calculated from a calibration curve obtained with chondroitin sulfate standard. For each sample type three samples were assayed. Results presented are the mean value and error bars corresponds to standard deviation.

\section{Total deoxyribonucleic acid}

The total deoxyribonucleic acid (DNA) present in the samples was determined using P7589 Quant-iT Picogreen dsDNA assay kit (Invitrogen) following the supplier's instructions.

In brief, the samples were prepared as for sGAG determination (digestion with proteinase $\mathrm{K}$ ) to free all DNA and protect it from DNAases released from lysed cells. Six standard DNA solutions with known concentrations were prepared for calibration. Aliquots of samples and standards were mixed with Picogreen Working Solution and corresponding quantity of assay Buffer. After reaction for $5 \mathrm{~min}$ in the dark at room temperature, fluorescence was read on a Victor microplate reader (Perkin Elmer) in triplicate. DNA concentration was determined using the calibration curve. For each sample type three samples were assayed. Results presented are the mean value and error bars corresponds to standard deviation.

\section{RESULTS}

\section{Scaffold and coating morphology}

As a result of the preparation process, scaffolds with high porosity $(86.6 \%$ as determined by the weight increase when the pore structure is filled with ethanol ${ }^{22}$ ) and double pore size distribution were obtained [Fig. 1(a)]. The macropores are produced as a result of the leaching of porogen particles out of the matrix, and their mean size is between 200 and $300 \mu \mathrm{m}$. Micropores are formed as a result of the solidliquid phase separation between dioxane and polycaprolactone during the freezing process. As can be seen in Figure $1(\mathrm{a})$, the structure is highly interconnected so as to permit colonization by cells and free diffusion of nutrients as well as metabolism by-products. The coating techniques succeed in producing two different coating morphologies. One step filling and crosslinking of the scaffold pores produces a HA gel phase that fills the macropores. When the swollen hydrogel is frozen and water sublimated in the CryoSEM a micropore structure is shown by this gel which is quite similar to that of the pore walls of PCL scaffold. The arrows in Figure 1(b) indicate the HA structure filling one of the 


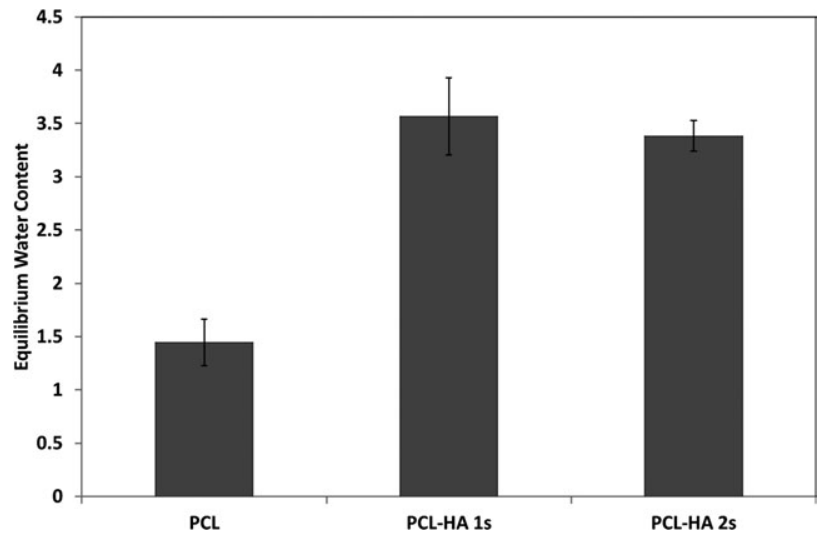

FIGURE 2. Equilibrium water content in PCL and PCL-HA samples. Error bars represent standard deviation.

macropores. After coating in two steps HA only covers the pore walls, sometimes hiding the microporosity [white arrows in Fig. 1(c)] while leaving the macropore space empty.

\section{Hyaluronic acid content and water uptake}

As a result of thermogravimetric analysis, it was determined that PCL-HA1s contained $4.6 \% \pm 2.3 \%$ and PCL-HA2s $5.8 \% \pm 2.8 \%$ hyaluronic acid by weight. The amount of hyaluronic acid is not significantly different depending on the coating procedures. As can be seen in Figure 2, HA coating leads to increased equilibrium water content, with a water uptake of nearly 3.5 for both samples regardless of the coating type $(3.6 \pm 0.4$ for PCL-HA1s and $3.4 \pm 0.1$ for PCLHA2s).

\section{Compression properties}

Compressive elastic modulus $E$ of the samples is not significantly influenced by the modification with $\mathrm{HA}$ as can be seen in Figure 3, although PCL-HA1s have higher mean stiffness, probably due to the filling of the pores. The only significant difference in seen between wet PCL-HA1s and wet PCL-HA2s. In general all wet samples have lower mean moduli.

\section{Immunofluorescent staining}

In Figures 4 and 5, light microscopy pictures of the construct slices $(100 \mu \mathrm{m})$ have been combined with the immunofluorescent pictures from confocal laser scanning microscope to allow for simultaneous visualization of the cells and the construct structure. Scaffold material appears as black, pore space as gray; in PCL-HA1s samples, the HA phase appears as translucid fibers that cross the pore space. In Figure 4 constructs are marked for collagen I and II, in Figure 5 for aggrecan and actin cytosqueleton. Cell distribution is different depending on the material type. Whereas in bare PCL cells are very homogenously distributed, in PCLHA1s (and to a less extent in PCL-HA2s) cells tend to form aggregates. In general, in HA containing samples, cells tend to grow in three dimensions, whereas in bare PCL scaffold, except in a few cases, cells are spread on the pore walls, and grow in volume only after locally reaching confluency. In PCL-HA1s, different areas of the sample cells show diverse behavior, whereas in control samples and PCL-HA2s the behavior is more homogenous. All samples show markers of collagen I and II. For instance, in bare PCL collagen I and II are localized in the same parts of the construct and appear mostly intracellularly (Fig. 4). In HA-coated samples there are domains with predominance of either one collagen type or the other, localized in different parts; in general, collagen type II is predominant in the areas of high cell density and cell aggregates, whereas collagen type I is predominant in the cells that are directly spread on the pore walls (also see Supporting Information Fig. 4). The only exception to this behavior is seen in PCL-HA2s at 21 days, where collagen I and II appear in a homogenous manner within the pore space. Most collagen is intracellular, excepted for PCL-HA1s where some collagen deposition outside the cell is observed.

Developed actin cytoskeleton is seen for an overwhelming majority of cells in all constructs; cells are generally spread on the pore surface or crossing the pore space; nevertheless, in PCL-HA1s, nearby the spread cells on pore walls, one can observe that in the cell clusters some cells lack the actin stress fiber network and their presence is only revealed by DAPI nucleus staining (see e.g., the two cells at the center of the picture at day 7).

As can be seen in Figure 5, there is nearly no presence of aggrecan in the PCL construct at any time. Aggrecan is most marked in PCL-HA1s samples; in these samples, it appears mainly in the cell aggregates although not every cell cluster is marked for SGAG as can be seen for day 14 (right side of the picture). Only in these samples aggrecan seems to appear out of the cells, while in PCL-HA2s, it is mostly stained within the cell bodies or close to nucleus. In some cases, the arrangement of the aggrecan as a sphere surrounding the cell resembles a pericellular matrix (same cells as mentioned before, day 7). In the case of the clusters unfortunately the cell density leads to very high signal

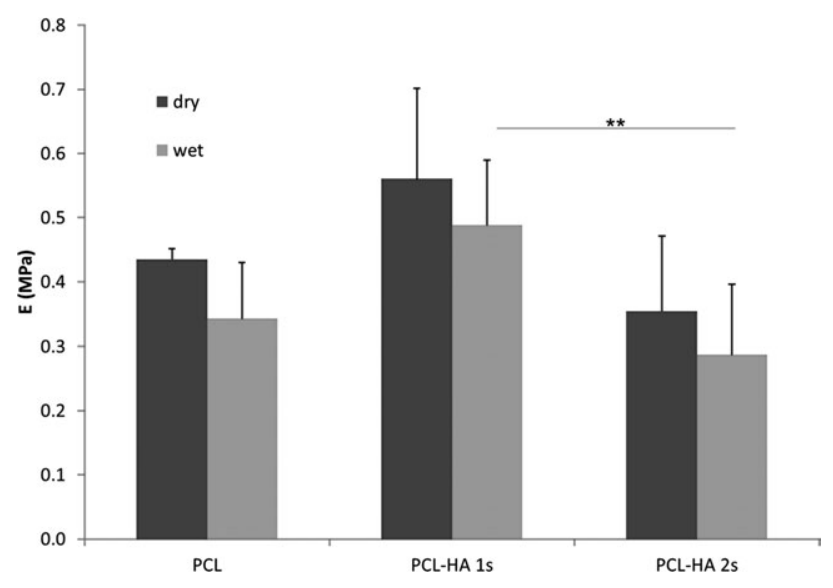

FIGURE 3. Elastic compressive modulus $(E)$ of the samples in dry and wet state. Error bars represent standard deviation. (Statistically significant differences are noted with ${ }^{*}$ for $p<0.05$ ). 


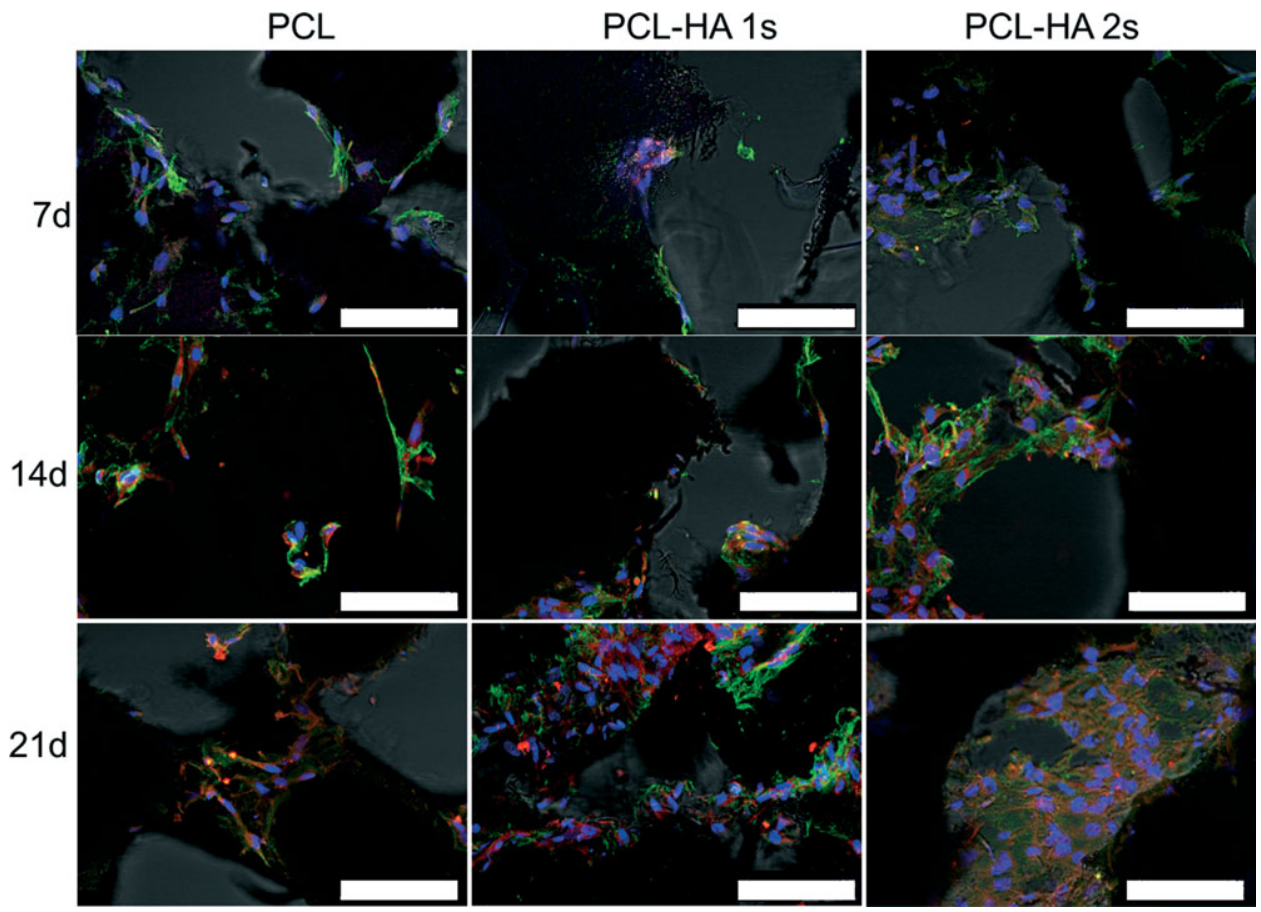

FIGURE 4. Composition of light microscopy and immunofluorescent staining for collagen I (green) and II (red) (scale bar $100 \mu \mathrm{m})$. The black areas of the pictures correspond to the scaffold. [Color figure can be viewed in the online issue, which is available at wileyonlinelibrary.com.]

intensity, making interpretation difficult. Pictures at larger scale are found in Supporting Information (Figs. 4 and 5).

As can be seen in Figure 6, staining for CD44 has very low intensity in PCL samples at 21 days, with many cells showing little or no expression of CD44. On the other hand, there is staining for CD44 in PCL-HA1s and PCL-HA2s samples in most cells of the constructs. This shows a higher
CD44 expression in cells that were cultured in the presence of hyaluronic acid.

\section{Quantitative biochemical assays}

The total DNA is always higher in bare PCL samples than in HA modified samples regardless of the type of modification (Fig. 7). To simplify the presentation of results, significant

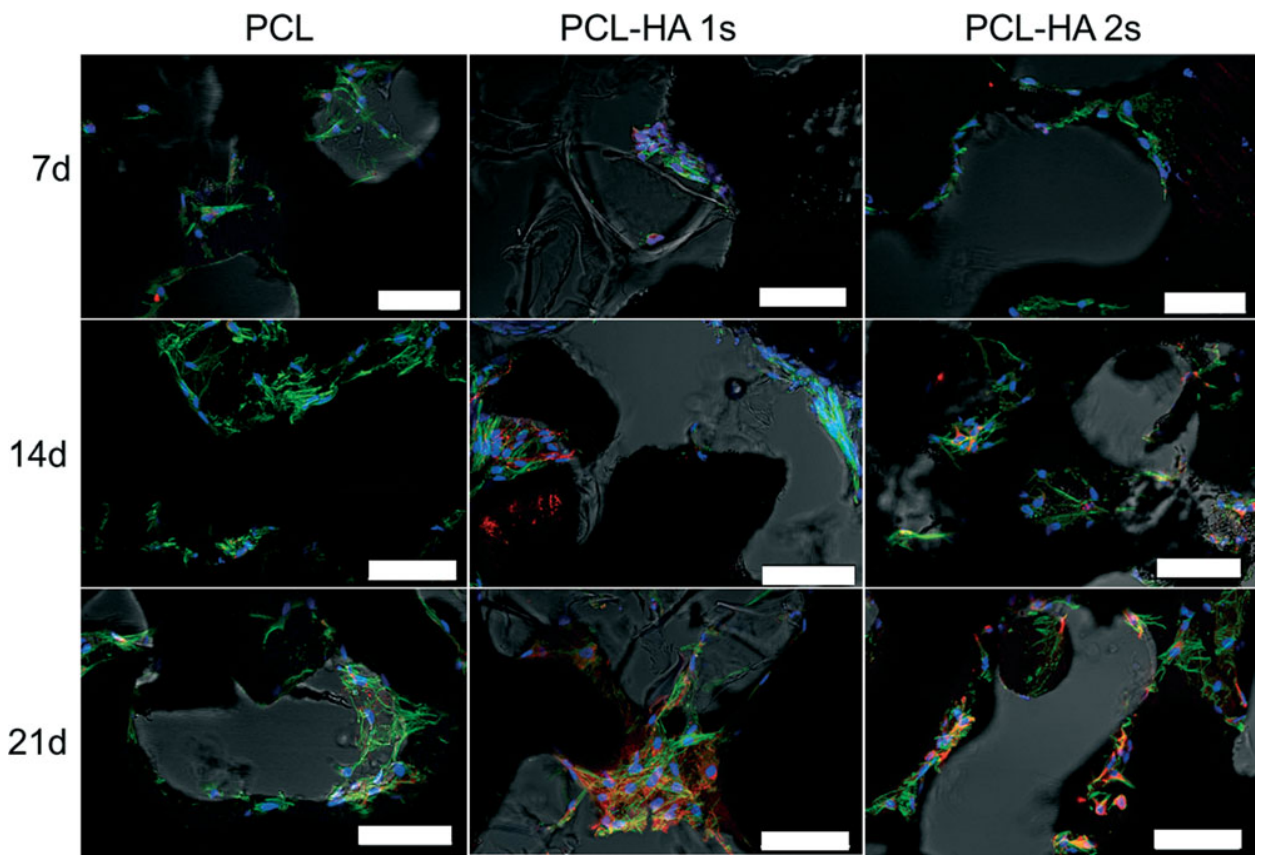

FIGURE 5. Immunofluorescent staining for proteoglycan (red) and actin (green) (scale bar $100 \mu \mathrm{m}$ ). [Color figure can be viewed in the online issue, which is available at wileyonlinelibrary.com.] 


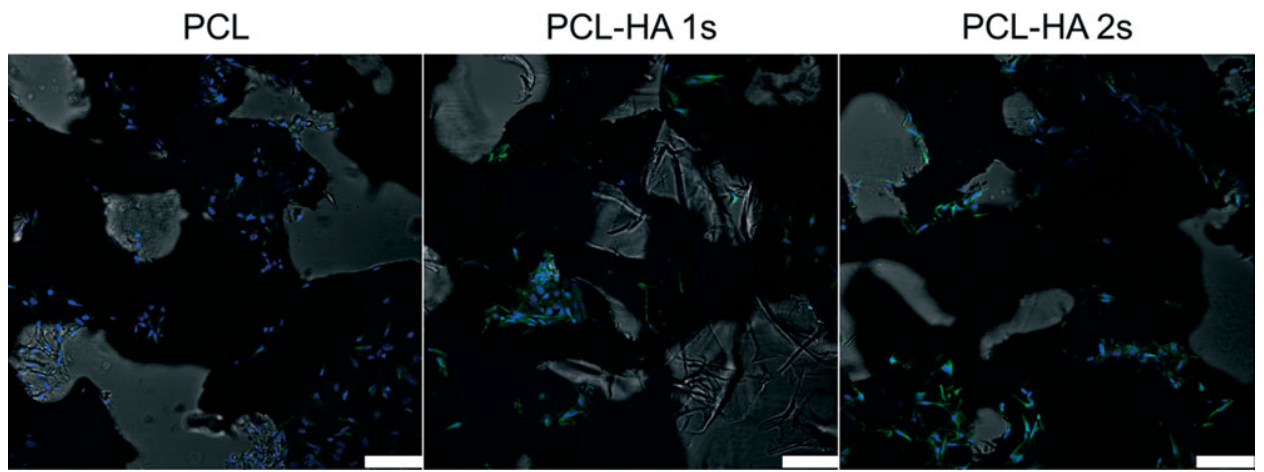

FIGURE 6. Inmunofluorescent staining for CD44 receptors (green) and nuclei (blue). [Color figure can be viewed in the online issue, which is available at wileyonlinelibrary.com.]

differences are only pointed out between different materials at the same time or between day 21 and day 7. There are some clues pointing towards a low seeding efficiency in PCL-HA scaffolds, particularly PCL-HA1s (Supporting Information Fig. 6).

In the case of PCL and PCL-HA1s, cell number grows with time, whereas in PCL-HA2s at 14 days there is a decrease in cell number and then cell number increases again at day 21 and becomes higher than in PCL-HA1s. All samples can be described as biocompatible and supporting cell adhesion and cell growth; no cytotoxicity due to DVS is observed. The proliferation ratio between day 21 and day 7 is highest for PCL-HA2s (2.4 \pm 0.5$)$, followed by PCL-HA1s $(1.9 \pm 1.7)$ (increase in cell number is not significant due to high dispersion); the minor ratio is observed for PCL (1.7 $\pm 0.6)$.

Sulfated glycosaminoglycan (sGAG) content in the constructs is presented in Figure 8. To simplify the presentation of results, significant differences are only noticed between different materials at the same time or between day 21 and day 7. As can be seen, sGAG absolute content is higher in PCL control samples than in other samples, (statistically significant at day 14 with $p<0.05$ ). Significant differences are found between day 21 and 7 for both PCL and PCL-HA2s

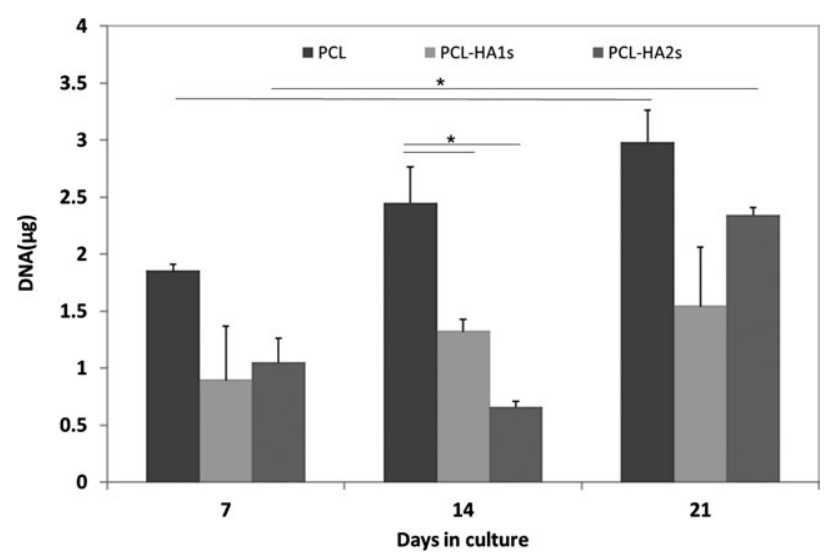

FIGURE 7. DNA content in the cultures. Error bars represent standard deviation. (Statistically significant differences are indicated by * for $p<0.05)$. samples $(p<0.05)$. Based on the observation that sGAG content follows roughly the tendency of DNA content in the samples, we decided to compare the ratio of sGAG to DNA as a qualification of the SGAG production per cell. Results are shown in Figure 9. As can be seen, at all times the glycosaminoglycan production per cell is significantly higher $(p<0.05)$ in PCL-HA1s than in bare PCL; sGAG content normalized to cell number is not statistically different at day 21 when compared to day 7 in none of the samples, although an increasing trend is seen in PCL-HA1s and decreasing trend in other samples. PCL-HA2s shows a similar trend to PCL, with no significant difference unless at day 14 where the sGAG content is significantly lower than in PCL. At day 21 the most sGAG per cell is found in PCL-HA 1step samples, with significant differences regarding PCL and PCL-HA2s $(p<0.05)$; this difference is not due to decreasing cell number in PCL-HA1s sample, as mean DNA content in PCL-HA1s samples increases with time as seen in Figure 7.

\section{DISCUSSION}

In this work we aimed to verify the hypothesis that modifying PCL scaffolds with HA could improve the three-dimensional substrates with respect to the proposed application, that is, articular cartilage tissue engineering. The effect of

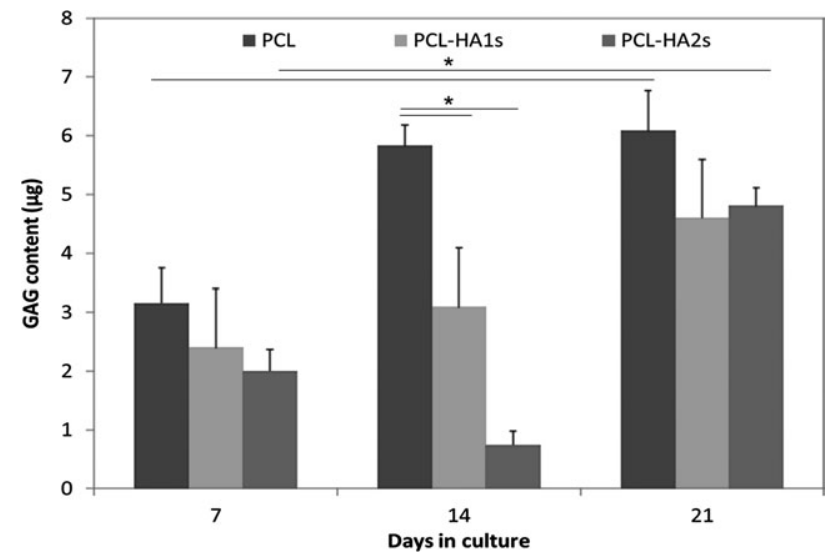

FIGURE 8. sGAG content [total, $(\mu \mathrm{g})$ ]. Error bars represent standard deviation. (Statistically significant differences are noted with $* *$ for $p<0.05)$. 


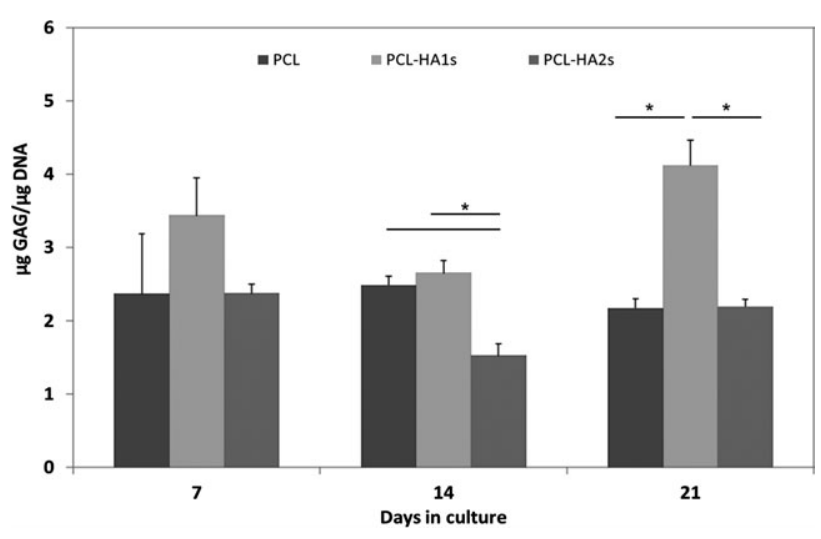

FIGURE 9. sGAG content normalized with respect to total DNA ( $\mu \mathrm{g}$ $\mu \mathrm{g}^{-1}$ ). Error bars represent standard deviation. (Statistically significant differences are noted with ** for $p<0.05)$.

HA on chondrocyte behavior was tested in unfavorable conditions where dedifferentiation is likely to occur due to the use of fetal bovine serum ${ }^{20}$ (which is employed to favor cell adhesion due to protein adsorption and to boost cell growth due to the presence of growth factors) ${ }^{24}$ and to the low cell densities used due to the use of human primary cell line.

As described in the results section, although both HAmodified samples contains similar amounts of HA, the incorporated HA shows a different microstructure depending on the methodology used, with homogenous crosslinking leading to a gel phase that fills the pores, whereas crosslinking of HA in two steps (PCL-HA2s) leads to a thin coating on the scaffolds' pore walls. Both samples are more hydrophilic than the bare PCL sample due to the presence of hyaluronic acid. When the hydrophobic PCL scaffold was immersed in liquid water, the swelling ratio was only around 1.4 (Fig. 2) as high hydrophobicity and surface tension impedes water penetration inside the scaffold, which can be a drawback when implanted in vivo. HA coating improves wettability and allows for water penetration inside the pore structure, regardless of the coating strategy used. Nevertheless, as seen by CryoSEM, microenvironment inside the hydrated scaffold is different, with presence of a gel phase in PCLHA1s and thin coating on the wall in PCL-HA2s, low swelling degree was observed in thin films made using the same two-step procedure (see Supporting Information Fig. 3) what explains that the HA coating in PCL-HA2s does not fill the pores when swollen.

Mechanical properties of the scaffolds tested were similar and show nearly no statistically significant differences. There were initially some concerns about the effect of treatment because of use of sodium hydroxide (which can cause a cleavage of the ester bonds). As can be seen in Figure 3 there is no statistically significant difference in the moduli in dry or wet state; the effect of the treatment on PCL scaffolds mechanical properties is minimal and should not compromise the further use of the scaffold as a chondral implant. In the wet state, modulus decreases as described by other groups even when testing polycaprolactone based materials. ${ }^{25,26}$ Despite the high hydrophobicity, water diffusion inside the amorphous and crystalline part of PCL is very fast, ${ }^{27}$ and may lower stiffness by decreasing interchain and intrachain interaction due to electrostatic interaction between water dipoles and carbonyl groups of PCL.

The elastic compressive moduli shown are in the range of moduli described for human articular cartilage by Athanasiou et al. $^{28}(0.5-1.82 \mathrm{MPa})$ although other groups have obtained higher values of $8-13.5 \mathrm{MPa}^{29}$ depending on the joint observed, when measuring with higher loading rate, described as "instantaneous modulus." In a previous work, we implanted macroporous PCL scaffolds (with similar moduli) in a rabbit knee $\operatorname{model}^{10}$; after 3 months, scaffolds seeded with allogenic chondrocytes were invaded by neoformed tissue; indentation measurements showed that the elastic modulus of the tissue-scaffold construct was the same than that of native cartilage controls (and significantly higher than pellet control). The values of the moduli measured here are similar and should grant adequate mechanical properties in vivo if the pore structure is successfully invaded by tissue. Higher initial modulus could be easily reached by varying the PCL content in the solution during the scaffold preparation (equilibrium modulus up to 4-5 $\mathrm{MPa}$ ). Sample stiffness is an important factor in articular cartilage tissue engineering as articular cartilage is subjected to high dynamic compression loading "in vivo." It has been shown that cartilage cells change their secretion profile $^{30}$ when exposed to an excessive load: catabolic activity becomes faster than anabolic activity, which provokes a loss of proteoglycans, ${ }^{31}$ matrix degradation and consequently a further decrease in mechanical properties. This usually leads to a repair tissue with inferior biochemical and biomechanical properties. ${ }^{8}$ Matching rigidity of cartilage tissue using only a hydrogel is very difficult, and moreover the high chain density and network crosslinking necessary to obtain such rigidities using hydrogels has been shown to inhibit ECM production. ${ }^{32}$ This type of structure (particularly PCL-HA1s) is thus interesting as the polyester scaffolding guarantees adequate macroscopic mechanical behavior, yielding a rigidity similar to cartilage while the microenvironment as sensed by the cells is a kind of dilute jelly, thus favoring cell migration, ECM production and diffusion.

Cells adhered to the material and proliferated in all materials. Seeding efficiency was lower in PCL-HA1s, which could be related to the presence of gel phase inside the pores as seen in Figure 1(b). This explains also the low densities found at day 7 in confocal microscopy; heterogeneous cell distribution may be due to limited diffusion throughout the gel. HA may also be responsible for reduced initial adhesion and seeding efficiency due to its hydrophilic character that does not permit non-specific adsorption of proteins. ${ }^{11}$ As shown in the confocal microscopy images, the first and obvious differences in chondrocyte behavior is the cell arrangement within the scaffold. Whereas in PCL scaffolds the cells tend to grow scattered on the pore walls, in presence of hyaluronic acid cells are seen filling the pore space, in case of PCL-HA1s with less homogeneous distribution and cell clustering. It has been described in literature that scaffolding materials, although presenting a 3D structure, do not necessarily encourage 3D growth in vitro, and that the behavior in scaffolds may be very similar to that of 2D cultures 
if at the cell's scale, the pore wall appears as a flat surface. ${ }^{33}$ This fact has motivated the use of cell carrier together with scaffolds, ${ }^{34}$ or secondary gel phases inside the pores, ${ }^{35}$ to get a three-dimensional tissue growth as occurs in vivo mainly due to the formation of a fibrin clot inside the implant. Here it appears that the modification of PCL with a hydrogel, in this case HA, can change this behavior, although it is not clear why. Lower modulus of HA may allow for greater mobility of cells; it is known that on materials with rigidity gradients, cells tend to accumulate on the stiffer parts, ${ }^{36}$ and that focal adhesions are strengthened by the increased rigidity. ${ }^{37}$ As a matter of fact, formation of marked stress fibers in the actin cytoskeleton, which is associated with dedifferentiated phenotype, ${ }^{38,39}$ is more prominent in PCL control than in other samples. This difference in growth mode may account for some of the effects observed in the immunofluorescent marking for collagen I, II and aggrecan. Another clue feature may be the activation of intracellular pathways due to the binding of cells to hyaluronic acid through CD44; as a matter of fact CD44 expression at the end of culture time is higher in samples that contain HA, regardless of the structure of HA in the samples (Fig. 6). High levels of CD44 expression have been described as a clue for increased chondrogenic capacity in chondrocytes subpopulations and high expression of CD44 and integrin $\alpha 3$ was associated with more GAG production per cell and more m-RNA of collagen type II. ${ }^{40}$

The formation of a pericellular matrix like coating marked for aggrecan observed occasionally in PCL-HA1s samples may be triggered by the interaction of hyaluronic acid with CD44 and to the better retention of small proteoglycans due to constructive interaction with exogenous hyaluronic acid at specific binding sites. ${ }^{41}$ Both in PCL-HA1s and PCL-HA2s, molecular weights between crosslinks are far higher than the number of HA saccharides necessary for CD44 binding (observed already with hexasaccharides). ${ }^{42}$ Nevertheless intracellular cascades that depend on CD44 may react in a different way upon binding; for instance clustering of CD44 with other membrane components may be hampered if $\mathrm{CD} 44$ receptor is anchored to HA chain with very low mobility and high stiffness, as may be the case in PCL-HA2s. This may explain why there are scarce quantitative differences observed between PCL-HA2s samples and PCL controls despite the qualitative differences observed by immunofluorescent staining. Another mechanism implied in the different response in PCL-HA1s and PCL-HA2s could be the influence of molecular crowding and of HA chain mobility on behavior, that is, ECM synthesis. ${ }^{43}$ It is also likely that albeit due to restricted diffusion through the gel phase or due to an enhancement in the specific interaction between aggrecan and HA binding sites due to chain mobility sGAG retention inside the construct is easier in PCL-HA1s. Higher normalized SGAG content could be due to better retention or to higher synthesis of sGAG; quantitative analysis of gene expression or analysis of sGAG secreted in the medium could help clarify this point in further studies.

\section{CONCLUSIONS}

Primary articular chondrocytes were cultured in dedifferentiating conditions and the effect of two types of hyaluronic acid coating on cell proliferation, cell morphology, sGAG synthesis, ECM and cell markers was studied. Cells in PCL controls show signs of dedifferentiation as reduced biosynthetic capacity, low staining for collagen type II and aggrecan and increased staining for collagen type I. In control samples the cells grow stuck on the pores walls, cells show a fibroblastic shape, and their behavior can be assimilated to 2D behavior. In samples modified with HA, cell distribution is more heterogeneous and different cell subpopulations are found in the construct, with the formation of cell clusters that depending on localization or cell organization show either positive markers for collagen type I or collagen type II and aggrecan. In some zones behavior is similar to that observed in PCL control (cells spread on pore wall), while in other zones formation of cell clusters and three dimensional growth is observed; in these zones there is more presence of cartilage specific ECM like COL II and aggrecan. In both HA containing samples, markers for CD44 are detected on most cells whereas in pure PCL samples there is hardly any presence of CD44. ECM production per cell is higher in PCLHA1s than in both PCL and PCL-HA2s samples.

All the mentioned results point towards a better phenotypic conservation in PCL-HA samples, with promising results when using PCL-HA1s. Enhanced hydrophilicity of the constructs and increased CD44 expression of chondrocytes in presence of HA shows that the strategy followed could be useful for cartilage tissue engineering in a cell-free approach if the increased hydrophilicity favors cell invasion and the presence of HA permits to home CD44 positive cells, which will be the subject of future works.

\section{ACKNOWLEDGMENTS}

The authors thank the microscopy service of Universidad Politécnica de Valencia as well as the confocal microscopy service of the Research Center Principe Felipe for useful help and advice. They thank Dr. Ignacio Muñoz from Clinica de la Salud for the production of primary chondrocyte population and Cristina Martinez for help with immunostaining protocols. None of the authors have any conflict of interest to declare.

\section{REFERENCES}

1. Van der Kraan PM, Buma P, Van Kuppevelt T, Van Den Berg WB. Interaction of chondrocytes, extracellular matrix and growth factors: Relevance for articular cartilage tissue engineering. Osteoarthritis Cartilage 2002;10:631-637.

2. Brittberg $M$, Lindahl $A$. Tissue engineering of cartilage. In: Clemens van Blitterswijk, editor. Tissue Engineering. Chapter 18. $p$ 533559. Elsevier/Academic Press, Amsterdam 2008.

3. Cancedda R, Dozin B, Giannoni P, Quarto R. Tissue engineering and cell therapy of cartilage and bone. Matrix Biol 2003;22:81-91.

4. Schnabel M, Marlovit S, Eckhoff G, Fichtel I, Gotzen I, Vecsei V, Schlegel J. Dedifferentiation-associated changes in morphology and gene expression in primary human articular chondrocytes in cell culture. Osteoarthritis Cartilage 2002;10:62-70.

5. Brodkin KR, García AJ, Levenston ME. Chondrocyte phenotypes on different extracellular matrix monolayers. Biomaterials 2004; 25:5929-5938.

6. Darling EM, Athanasiou KA. Rapid phenotypic changes in passaged articular chondrocyte subpopulations. J Orthoped Res 2005;23:425-432.

7. Nehrer S, Domayer S, Dorotka R, Schatz K, Bindreiter U, Kotz R. Three-year clinical outcome after chondrocyte transplantation 
using a hyaluronan matrix for cartilage repair. Eur J Radiol 2006; 57:3-8.

8. Vinatier C, Mrugala D, Jorgensen C, Guicheux J, Noël D. Cartilage engineering: A crucial combination of cells, biomaterials and biofactors. Trends Biotechnol 2009;27:307-314.

9. Solchaga LA, Temenoff JS, Gao J, Mikos AG, Caplan Al, Goldberg VM. Repair of osteochondral defects with hyaluronan- and polyester-based scaffolds. Osteoarthritis Cartilage 2005;13:297-309.

10. Martinez-Diaz S, Garcia-Giralt N, Lebourg M, Gomez Tejedor JA Vila G, Caceres EB, Pedro Nogues X, Gomez Ribelles JL, Monllau JC. In vivo evaluation of three-dimensional poly( $\varepsilon$-caprolactone) scaffolds for cartilage repair in rabbits. Am J Sport Med 2010;38: 509-519.

11. Bonzani IC, George JH, Stevens MM. Novel materials for bone and cartilage regeneration. Curr Opin Chem Biol 2006;10:568-575.

12. Schagemann JC, Kurz H, Casper ME, Stone JS, Dadsetan M, YuLong S, Mrosek EH, Fitzsimmons JS, O'Driscoll SW, Reinholz GG. The effect of scaffold composition on the early structural characteristics of chondrocytes and expression of adhesion molecules. Biomaterials 2010;31:2798-2780.

13. Patti AM, Gabriele A, Vulcano A, Ramieri MT, Della Rocca C. Effect of hyaluronic acid on human chondrocyte cell lines from articular cartilage. Tissue Cell 2001;33:294-300.

14. Yoo HS, Lee EA, Yoon JJ, Park TG. Hyaluronic acid modified biodegradable scaffolds for cartilage tissue engineering. Biomaterials 2005;26:1925-1933.

15. Grigolo B, De Franceschi L, Roseti L, Cattini L, Facchini A. Down regulation of degenerative cartilage molecules in chondrocytes grown on a hyaluronan-based scaffold. Biomaterials 2005;26: 5668-5676

16. Girotto D, Urbani S, Brun P, Renier D, Barbucci R, Abatangelo G Tissue-specific gene expression in chondrocytes grown on threedimensional hyaluronic acid scaffolds. Biomaterials 2003;24 3265-3275.

17. Brun P, Panfilo S, Gordini D, Cortivo R, Abatangelo G. The effect of hyaluronan on CD44-mediated survival of normal and hydroxyl radical-damaged chondrocytes. Osteoarthritis Cartilage 2003;11: 208-216.

18. Sharma B, Elisseeff JH. Engineering structurally organized cartilage and bone tissues. Ann Biomed Eng 2004;32:148-159.

19. Antunes JC, Oliveira JM, Reis RL, Soria JM, Gómez Ribelles JL, Mano JF. Novel poly(L-lactic acid)/hyaluronic acid macroporous hybrid scaffolds: Characterization and assessment of cytotoxicity. J Biomed Mater Res 2010;94A:856-869.

20. Garcia-Giralt N, Izquierdo $R$, Nogués $X$, Perez-OImedilla $M$, Benito P, Gomez-Ribelles JL, Checa MA, Suay J, Caceres E, Monllau JC. A porous $P C L$ scaffold promotes the human chondrocytes redifferentiation and hyaline-specific extracellular matrix protein synthesis. J Biomed Mater Res 2008;85A:1082-1089.

21. Ho MH, Kuo PY, Hsieh HJ, Hsien TY, Hou LT, Lai JY, Wang DM. Preparation of porous scaffolds by using freeze-extraction and freeze-gelation methods. Biomaterials 2004;25:129-138.

22. Lebourg M, Suay Anton J, Gomez Ribelles JL. Hybrid structure in PCL-HAp scaffold resulting from biomimetic apatite growth. J Mater Sci Mater Med 2010;21:33-44.

23. Collins $M N$, Birkinshaw C. Comparison of the effectiveness of four different crosslinking agents with hyaluronic acid hydroge films for tissue-culture applications. J Appl Polym Sci 2007;104: 3183-3191.

24. Freyria $A M$, Cortial D, Ronzière MC, Guerret S, Herbage D. Influence of medium composition, static and stirred conditions on the proliferation of and matrixprotein expression of bovine articular chondrocytes cultured in a 3D collagen scaffold. Biomaterials 2004;25:687-697.
25. Wang Y, Liu L, Guo S. Characterization of biodegradable and cytocompatible nano-hydroxyapatite/polycaprolactone porous scaffolds in degradation in vitro. Polym Degrad Stab 2010;95: 207-213.

26. Pok SW, Wallace KN, Madihally SV. In vitro characterization of polycaprolactone matrices generated in aqueous media. Acta Biomater 2010;6:1061-1068.

27. Harrison $\mathrm{KL}$, Jenkins MJ. The effect of crystallinity and water absorption on the dynamic mechanical relaxation behavior of polycaprolactone. Polym Int 2004;53:1298-1304.

28. Samarza AJ, Athanasiou A. Design characteristics for the tissue engineering of cartilaginous tissues. Ann Biomed Eng 2004;32: 2-17.

29. Shepherd DET, Seedhom BB. The instantaneous compressive modulus of human articular cartilage in joints of the lower limb. Rheumatology 1999;38:124-132.

30. Quinn TM, Grodzinsky AJ, Buschmann MD, Kim YJ, Hunziker EB. Mechanical compression alters proteoglycan deposition and matrix deformation around individual cells in cartilage explants. J Cell Sci 1998;111:573-583.

31. Grodzinsky AJ, Levenston ME, Jin M, Frank EH. Cartilage tissue remodeling in response to mechanical forces. Annu Rev Biomed Eng 2000;2:691-713.

32. Chung C, Burdick JA. Engineering cartilage tissue. Adv Drug Deliv Rev 2008;60:243-262.

33. Nuernberger S, Cyran N, Albrecht C, Redl H, Vécsei V, Marlovits $\mathrm{S}$. The influence of scaffold architecture on chondrocyte distribution and behavior in matrix-associated chondrocyte transplantation grafts. Biomaterials 2011;32:1032-1040.

34. Hutmacher DW, Ng KW, Kaps C, Sittinger M, Klaring S. Elastic cartilage engineering using novel scaffold architectures in combination with a biomimetic cell carrier. Biomaterials 2003;24: 4445-4458.

35. Sato T, Chen G, Ushida T, Ishii T, Ochiai N, Tateishi T, Tanaka J. Evaluation of PLLA-collagen hybrid sponge as a scaffold for cartilage tissue engineering. J Mater Sci Eng C 2004;24:365-372.

36. Discher DE, Janmey $P$, Wang $Y$. Tissue cells feel and respond to the stiffness of their substrate. Science 2005;310:1139-1143.

37. Choquet D, Felsenfeld DP, Sheetz MP. Extracellular matrix rigidity causes strengthening of integrin-cytoskeleton linkages. Cell 1997; 88:39-48.

38. Woods A, Wang G, Beier F. Regulation of chondrocyte differentiation by the actin cytoskeleton and adhesive interactions. J Cell Physiol 2007;213:1-8.

39. Garcia-Giralt N, Izquierdo R, Nogués $X$, Perez-Olmedilla M, Benito P, Gomez-Ribelles JL, Checa MA, Suay J, Caceres E, Monllau JC. A porous PCL scaffold promotes the human chondrocytes redifferentiation and hyaline-specific extracellular matrix protein synthesis. J Biomed Mater Res 2008;85A:1082-1089.

40. Grogan SP, Barbero A, Diaz-Romero J, Cleton-Jansen AM, Soeder S, Whiteside R, Hogendoorn PCW, Farhadi J, Aigner T, Martin I, Mainil-Varlet P. Identification of markers to characterize and sort human articular chondrocytes with enhanced in vitro chondrogenic capacity. Arthritis Rheum 2007;56:586-595.

41. Knudson CB. Hyaluronan and CD44: Strategic players for cell-matrix interactions during chondrogenesis and matrix assembly. Birth Defects Res C 2003;69:174-196.

42. Stern R, Asari AR, Sugahara KN. Hyaluronan fragments: An information rich system. Eur J Cell Biol 2006;85:699-715

43. Lareu RR, Subramhanya KH, Peng $Y$, Benny $P$, Chen $C$, Wang $Z$, Rajagopalan $R$, Raghunath $M$. Collagen matrix deposition is dramatically enhanced in vitro when crowded with charged macromolecules: The biological relevance of the excluded volume effect. FEBS Lett 2007;581:2709-2714. 